\title{
The increasing of energy efficiency of international freight truck trains under the concept of energy innovative technologies development motor transport
}

\author{
Tatiana Kostiuk ${ }^{*}$, and Ramasan Habutdinov \\ National Transport University, Kiev, Ukraine
}

\begin{abstract}
At the motor transport (MT) the concept of technological and innovative energy saving is actual. However, the existing method of increasing the cost-effective efficiency of motor transport does not correspond to this concept because the basis of the theory of the motor transport process consists of the axioms, which neglected: a) the fragmenting effect of existing technological paradoxes on the integrity of knowledge about motor transport as a sphere of material production; b) the resource, production and technological essence of this process; c) innovative variability of parameters of new cars and transport technologies. In addition, the basis of the fundamental designed scheme of the transport operation is based on simplified mathematical description of the virtual transposition operations. These methodological shortcomings do not allow to analyze and substantiate innovative technical and technological projects of motor transport. For the possibility of realization of the actual concept of technological and innovative energy saving on MT, in NTU the theory and methods of a comprehensive increase of energy efficiency of a car (EREC) of a general type, as well as methods of simulation and test formation of energy saving of motor transport technologies were designed.
\end{abstract}

\section{Formulation of the problem}

The main concept of the development of motor transport and motor transport system (MTS) is to ensure the technological development of their components on the basis of the conceptual idea of innovative operational and technological energy- and resource-saving (IOTERS) in all transport enterprises. In connection to this, the system methodology IOTERS is actual, on the basis of which it would be possible to form mutually coherent and harmonized methods of ensuring IOTERS in three main components of motor transport: the controlling substructure of transport (CST), resource and technological base (RTB), transport and technological processes (TTP). Due to the fact that existing theories of economics of the organization of transport processes have significant drawbacks, and most importantly, it is non-technological, fragmentary, non-technical, nontechnological, and does not take into account important processes energy transformation of resources into a tangible product of transport, so the theory of energy and energy type was developed. An epistemological obstacle to the coordination and harmonization of IOTERS methods for the mentioned components of motor transport is the methodological contradiction between the knowledge bases of specialists of the CST: organizers, transport managers and engineers [1]. This contradiction in many respects is stipulated due to the objective manifestations of six technological paradoxes
(TP) of transport, as spheres of material The subset of TP paradoxes of vehicles is written as follows:

$$
T P \in(T P 1, T P 2, T P 3, T P 4, T P 5, T P 6)
$$

where TP1- a paradox of the form of transport product (product is material, but not real); TP2-paradox of the method of product realization (synchronous creation and consumption); TP3 is a paradox of the form of spatialpoint dislocation of transport operations (transport operations are distributed in trajectory space of road network infrastructure, and terminal operations are carried out at certain points of the trajectory-in terminals); TP4- paradox of machine procedures of transport technologies (indirect and reactive character of machine actions on the subject of transportation; at the same intensity levels, these actions can be both productive-useful and trajectory-dangerous depending on the type of locally trajectory situations); TP5 is a substantive and valuable paradox of the processes of transformation of transport resources and transport production (the substance of resources is not transferred, and the cost of resources is transferred to the product). It should be noted that the fifth paradox violates the adequacy of the ratio of such product characteristics as "price-quantity", "price-quality." These ratios depend on the operational and technological conditions of transportation, which are not taken into account in the organizational theory of the transport process $[1,3]$.

*orresponding author: tani4ka.kostyuk@gmail.com 
TP6- a paradox of the form of the use of regime resources (the regime resources are converted into energy-driven time of the motor vehicle (MV)) and in this form is an important parameter of the transport operation and the physical product of transport.

The time of movement of MV on the local sections of the route of transportation is inversely proportional to the power function of the MV engine. It should be noted that this dependence is also not taken into account in the methods of organization of transportation [3]. Objective manifestations of paradoxes, on the one hand, lead to significant differences in the process of transport production from other spheres of material production.

On the other hand, the paradoxes lead to the discontinuity of the knowledge of different specialists of the CST. Firstly, organizers and economists use the simplified, techno-empirical and cost-effective methodology of transport services, which is based on the principle of unchangeability of technology and technology. Secondly, in the methodology the laws of the transposition process are considered but not transport. Thirdly, the properties of technical resources and the processes of their transformation into a transport product are ignored. In connection with the stated above, the existing methodology of transport services is: technoempirical, non-technological, non-resourceful and antiinnovation (the principle of unchangeability of technology and technology). As a result the FUT (freezing undescribed technology) is used. It does not allow to realize the concept of innovation and technological development of transport processes and vehicles [3] and new DDT (described developed technology) principle [4], [5]. The indicated shortcomings of the existing methodology lead to the most negative consequences for the domestic transport companies which are operating in the field of international transportation. In the international road transport services markets the requirements for future energy efficiency and technical and technological competitiveness of transport proposals are more stringent.

\section{Objectives and goals}

The main goal and objective of the article is the analysis of transport energy efficiency of international freight truck trains with detailed consideration of the influence of structural, technical, road, operational factors of the transport process. The article presents the method of simulation analysis of energy efficiency of international road trains as resource - technical means of transport production and forecasting their suitability for energy saving technologies. This technique allows simulating the energy efficiency of the TTP and is an important component of the IOTERS methodology.

\section{Main Part}

The researched works concerning efficiency on transport are actual and taken into account [3], [6], [8] however in the theory of transport process only the cost-effective efficiency is considered, without taking into account technical and technological innovations. In addition, it should be pointed out that motor transport is fundamentally different from other modes of transport, due to suchfactors as high structural diversity, a wide range of changes in road and terminal conditions of transportation, resizing party masses and trajectoryconflict infrastructure. In works [1,2,7] it was established that the tasks of innovation and technological development of motor transport need to be solved on the basis of a new paradigm of the theory of management on transport - material, productive and innovative. The above papers present the main provisions of this new paradigm, as well as four corresponding conclusions. Firstly, the epistemological basis for the development of the IOTERS methodology, taking into account the subset of technological paradoxes (1) is the theory of energyefficiency efficiency of the MT (EEMT) of the generalized (modular) type [4]. Secondly, the usefulness of the TTP for consumers of transport services is the result of the mass implementation of the processes of technological transportation of goods and passengers (TTGP). Each such process is a consequence of the driven process of ERW- the process of transformation technologic resources of transport into the physical transport product that is created under the given conditions. Thirdly, the tasks of innovative technological development of the TTP should be solved on the basis of the method of benchmarking - comparative analysis and forecasting of transport efficiency of new MV in test operations [4]. Fourthly, in order to create energy-saving transport technologies (TTs), it is necessary to solve the tasks of increasing energy efficiency of transportation: new MV, procedures of machine operations, processes of energy conversion MV and ERW.

The indicated tasks can not be solved using the known performance indicators of MV productivity and the cost of transportation due to the above mentioned methodological disadvantages [3]. Mathematical models of these indicators are based on the principle of invariance of technologies and on the calculation scheme of the transposition operation [3]. In regard to this, the indicated indicators are unsuitable for the improvement of transport technologies, and the model of the cost of transportation is anti-innovation. In work [4] it was established that the idea of improving transport technologies is a gradual increase in the value of the energy efficiency indicator (in a specific case - fuel consumption indicator) of transportation and this this the new approach in transport technology development of the motor transportation. To realize this idea, when substantiating the new rolling stock, the dimensionless indicator of the transport energy efficiency of the MV is used. Its value is determined by the formula

$$
I e=\frac{C_{h t}}{R_{I e}}
$$

where $C_{h t}$ - heat transfer coefficient of the new MV in the calculation route, which takes into account the energy efficiency of the new MV in test operations 
(urban, trunk, mixed and benchmark), the estimated share of MV in urban conditions and distribution of MV runway according to road categories [4].

$R_{l e}$ - the reference value of the index of energy efficiency of a car.

On the basis of formula (2) the following calculation formula is received:

$$
I_{e}=\frac{c_{a s c r} \cdot \gamma_{s t}}{c_{f m}\left(c_{r w}+\gamma_{s t}\right)}
$$

where $c_{\text {ascr }}$ - the dimensionless coefficient of average speed of MV on the calculated route:

$$
c_{\text {ascr }}=\frac{S_{a}}{S_{c}}
$$

where $S_{a}$ - average speed of the MV on the calculated route;

$S_{c}$ - reference (constant) speed;

$\gamma_{s t}$ - coefficient of static use of load-carrying capacity;

$c_{f m}$ - dimensionless fuel coefficient of mileage of the $\mathrm{MV}$;

$c_{r w}-$ coefficient of running weight;

$$
c_{f m}=\frac{f_{c e}}{f_{c r}}
$$

where $f_{c e}$ - the amount of estimated fuel consumption of the $\mathrm{MV}$ in the calculated route, $f_{c r}$ - reference fuel consumption.

Calculated formulas for determining the coefficients speed of $c_{\text {sto }}$ and mileage $c_{\text {fmto }}$ in test operations are as follows:

$$
c_{\text {sto }}=\frac{a_{1}\left(a_{2}-a_{3} \cdot \psi_{a}\right)}{a_{4} \cdot t_{d}+a_{5}}
$$

where $a_{1}, a_{2}, a_{3}, a_{4} a_{5}-$ constant parameters, which are determined for test transport operations, taking into account the provided conditions of the MV movement and its constructive parameters;

$\psi_{a}$ - average value of the resistance of the road,

$t_{d}$-time of dispersal of the MV to $60 \mathrm{~km} / \mathrm{h}$. (in urban cycle) and up to $80 \mathrm{~km} / \mathrm{h}$. (in the trunk cycle), s.

The value of the speed factor of the MV on the calculated route is determined by the formula:

$$
c_{s c r}=c_{s u c} \cdot t_{u c}+c_{s t c} \cdot\left(1-t_{u c}\right)
$$

where $t_{u c}$-share of time of work of MV in urban conditions; $c_{s u c}$ and $c_{s t c}$ - the value of the coefficient for urban and trunk cycles, respectively.

The value of the coefficient of fuel mileage in test operations is determined as follows:

$$
c_{\text {fmto }}=\frac{b_{1} \cdot\left(b_{2} \cdot \psi+b_{3}\right) \cdot c_{r t} \cdot F \cdot \rho}{b_{4} \cdot C \cdot\left(\gamma_{s t}+c_{r w}\right)+b_{5} \cdot H_{m v} \cdot W_{m v}}
$$

where $b_{1}, b_{2}, b_{3}, b_{4}, b_{5}$ - constant parameters, which are determined for test transport operations, taking into account the operating conditions of the MV and its constructive parameters.

$\psi$ - value of the resistance of the road,

$c_{r t}$ - coefficient of road traffic conditions;

$F$ - the total fuel consumption rate per $100 \mathrm{~km}$ of dispersal;

$\rho$-is the volume mass of fuel ( $\rho=0,76$ for gasoline;

$\rho=0,84$ for diesel fuel);

$C$ - load-carrying capacity of the MV, $\mathrm{t}$;

$\gamma_{s t}$ - coefficient of static use of load-carrying capacity;

$c_{r w}$ - coefficient of running weight;

$H_{m v}$ and $W_{m v}$ - height and width of the MV, m;

Fuel mileage factor on the calculated route:

$$
c_{f m c r}=c_{f m u c} \cdot t_{u c}+c_{f m t c} \cdot\left(1-t_{u c}\right)
$$

where $c_{f m u c}$ and $c_{f m t c}$ - the value of the coefficient for urban and trunk cycles;

$t_{u c}$ - share of time of ATZ in urban conditions.

We will analyze how the change of such constructive parameters of the road train as the wheel radius and the engine power will affect on the energy efficiency index . Our task is to choose from the existing range of cars models that ones, that will best meet our criteria and will be the most economical and this is the practical part of this theory.

Based on the calculated data, the diagrams of the dependences how the changes in wheel radius and engine power are influenced on the energy efficiency of the MT, such dependencies are presented in diagrams Fig. 1, 2. For existing freight truck trains, the power range changes from 360 to $600 \mathrm{~kW}$, wheel radius from 0.4 to $0.6 \mathrm{~m}$ according to the car's specifications.

On the basis of the multivariate calculation it was established that at a radius of $0.55 \mathrm{~m}$, the energy efficiency indicator $\left(I_{e}\right)$ reaches the maximum value, and when the engine power increases, the energy efficiency indicator decreases; the most optimal variants are with a power of $360 \mathrm{~kW}$. The quantitative characteristics of these laws are obtained.

On the basis of comparing the values of the energy efficiency indicator in Figures 1 and 2 it can be concluded that freight truck trains with a radius of wheel of $0.55 \mathrm{~m}$ are more in line with the IOTERS concept, since the energy efficiency indicatior is higher by 19.5 - 
$21.8 \%$, with the increase in engine power energy efficiency decreases by $18.1-25.3 \%$.

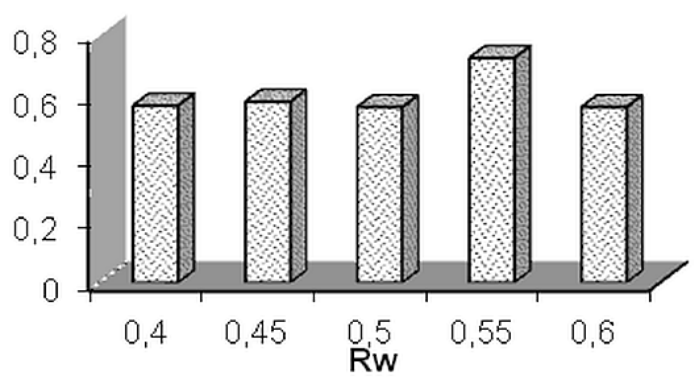

Fig. 1 - Diagram of the dependence of the energy efficiency index of the RT on the calculated route from the change in the radius of the wheel.

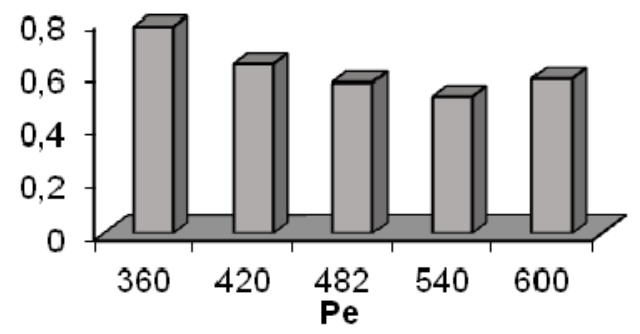

Fig. 2 - Diagram of the dependence of the energy efficiency index of the RT on the calculated route from the change in engine power.

Based on the conducted analysis, it is determined that, in contrast of calculations in the theory of the automobile, this technique takes into account several resource and technical properties (the car as a complex machine, the object of adaptive motion control in a multi-phase test operation, the car as a means of transport, etc., mathematical models adaptivelydiscrete kinematics and power engineering of the vehicle with variable parameters). Therefore, in practice, the desirability of operational choice and substantiation of the main parameters of freight truck trains taking into account the conditions of transportation is important.

\section{Conclusions}

1. It was discovered that the provision of technological development of motor transport is based on the conceptual idea of innovative operational and technological energy- and resource-saving (IOTERS) in all transport enterprises, because the existing methods do not allow to solve the problem of technological innovative development according to the concept of energy saving on transport

2. $t$ was revealed that for the implementation of the IOTERS concept, which allows a detailed account of technical and technological innovations, it is necessary to use mathematical models of the theory of energyresource efficiency of automobile.
3. The complex of mathematical models for the simulation and benchmarking analysis of energy efficiency of new MVs has been formed, which will enable the implementation of the concept of operational energy saving in all transport enterprises.

4. The technical characteristics of road truck trains are analyzed and quantitative characteristics of the influence of various technical factors on energy efficiency indicators are obtained.

5. The methodology of simulation and referencecomparative analysis of energy efficiency of new freight truck trains as resource and technical means of transport production is proposed. As a result, this methodology can be used at transport enterprises in order to improve energy efficiency of transportation and the introduction of new technologies, since it allows to select from the existing range of car models the ones that will best meet the theory of energy saving and will allow the introduction of innovations to improve the efficiency of freight transportation and the development of the transport enterprises at all.

\section{References}

1. R. Khabutdinov Transport technological paradigm and methodology of novation management of motorcar transportations. K.: Visnyk NTU, vol.24,part.2.,p. 237-240 (2011)

2. R. Khabutdinov Methodological bases of transporttechnological energology, Scientific papers "Problem of transport". K.: NTU, Vol. 3. P 164-168 (2006)

3. A.Vorkut Road transport of goods. K.: High-school, 447 p. (1986)

4. R. Khabutdinov, A. Kotsyк Energy-resourse efficiency of car. K.: UTU (1997)

5. R. Khabutdinov Systemic problem of innovative development of motor transport in conditions of theoretical denial of transport technologies and production. K.: Visnyk NTU, vol. 34, p. $491-496$ (2016)

6. H. Kravchenko The essence of the economic category of efficiency and definition in transport. D: The problems of transport economy, DNU, vol. 8, (2014)

7. R. Khabutdinov The methodology of conceptual and innovative management of technological development of vehicles. K.: Visnyk NTU, vol. 29, p. $409-414$ (2014)

8. P. Wang, R. Goverde Multi-train trajectory optimization for energy efficiency and delay recovery on single-track railway lines: Transportation Research Part B Methodological 105, pp. 340-361 (2017) https://doi.org/10.1016/j.trb.2017.09.012 